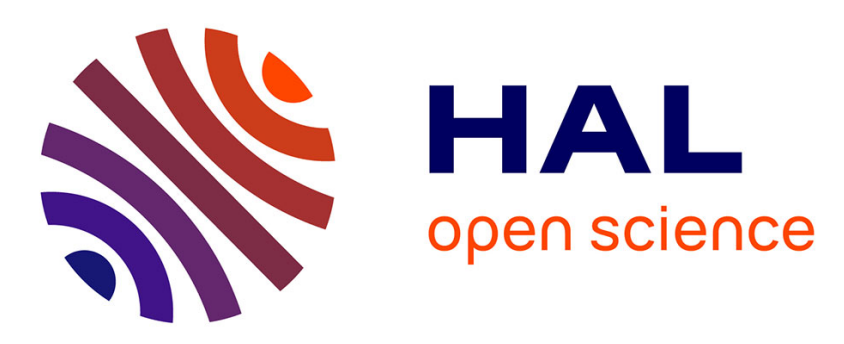

\title{
High Accuracy Volume Flow Rate Measurement Using Vortex Counting
}

Abdelkader Zaaraoui, Florent Ravelet, Florent Margnat, Sofiane Khelladi

\section{To cite this version:}

Abdelkader Zaaraoui, Florent Ravelet, Florent Margnat, Sofiane Khelladi. High Accuracy Volume Flow Rate Measurement Using Vortex Counting. Flow Measurement and Instrumentation, 2013, 33, pp.138-144. 10.1016/j.flowmeasinst.2013.06.002 . hal-00707329v4

\section{HAL Id: hal-00707329 \\ https://hal.science/hal-00707329v4}

Submitted on 17 Jun 2013

HAL is a multi-disciplinary open access archive for the deposit and dissemination of scientific research documents, whether they are published or not. The documents may come from teaching and research institutions in France or abroad, or from public or private research centers.
L'archive ouverte pluridisciplinaire HAL, est destinée au dépôt et à la diffusion de documents scientifiques de niveau recherche, publiés ou non, émanant des établissements d'enseignement et de recherche français ou étrangers, des laboratoires publics ou privés. 


\title{
High Accuracy Volume Flow Rate Measurement Using Vortex Counting
}

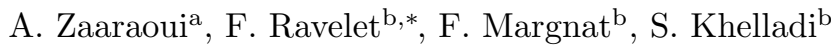 \\ ${ }^{a}$ Laboratoire Fluides Industriels Mesures et Applications, Université de Khemis Meliana, Algérie. \\ ${ }^{b}$ Arts et Metiers ParisTech, DynFluid lab., 151 boulevard de l'Hôpital, 75013 Paris, France.
}

\begin{abstract}
A prototype device for measuring the volumetric flow-rate by counting vortices has been designed and realized. It consists of a square-section pipe in which a two-dimensional bluff body and a strain gauge force sensor are placed. These two elements are separated from each other, unlike the majority of vortex apparatus currently available. The principle is based on the generation of a separated wake behind the bluff body. The volumetric flow-rate measurement is done by counting vortices using a flat plate placed in the wake and attached to the beam sensor. By optimizing the geometrical arrangement, the search for a significant signal has shown that it was possible to get a quasi-periodic signal, within a good range of flow rates so that its performances are well deduced. The repeatability of the value of the volume of fluid passed for every vortex shed is tested for a given flow and then the accuracy of the measuring device is determined. This quantity is the constant of the device and is called the digital volume $\left(V_{p}\right)$. It has the dimension of a volume and varies with the confinement of the flow and with the Reynolds number. Therefore, a dimensionless quantity is introduced, the reduced digital volume $\left(V_{r}\right)$ that takes into account the average speed in the contracted section downstream of the bluff body. The reduced digital volume is found to be independent of the confinement in a significant range of Reynolds numbers, which gives the device a good accuracy.
\end{abstract}

Keywords: Wake; Bluff body; Accuracy; Strain gauges sensor; Vortex shedding; Digital volume.

\section{Introduction}

Measurements of volumetric flow rates are common practices in fluid flow under pressure. The devices that are available and the corresponding techniques are of various types but generally guarantee a precision that rarely exceeds $1 \%$. However, the current needs of certain industries (petroleum, chemical etc...) go beyond that figure. To meet this demand, a solution is provided by methods of measurement based on counting the vortices generated in the wake of a bluff body in a two-dimensional flow.

In a free medium, for a perfect fluid, the wake downstream of a bluff body is characterized by the presence of two parallel vortex sheets where the vorticity is concentrated $[1,2]$. For a real fluid, the configuration depends on the Reynolds number, usually based on the free-stream velocity $v_{1}$ and a typical length of the cross-section of the bluff body $d$ (see figure 1 left). At very low Reynolds number (of the order of the unity) the flow is steady and presents all the symmetries of the problem. As the Reynolds number increases, the vortex sheets first give nice stable and periodic roller shaped vortices to form the "von Kármán vortex street" (see figure 1). For Reynolds numbers of the order of $10^{2}$, there is a unique frequency $f$ that is usually given in a dimensionless form with a Strouhal

\footnotetext{
* corresponding author

Email address: florent.ravelet@ensta.org (F. Ravelet)
}

number $S t=\frac{f \cdot d}{v_{1}}$. In this intermediate Reynolds numbers range, the Strouhal number varies with the Reynolds number monotonically. For large Reynolds number (in the range $10^{3}-10^{5}$ ) a wake with a certain periodicity is still observed in spite of the instabilities: the vortices now do not detach on a regular basis, but the frequency spectrum of the wake presents a peak at a well-defined frequency $f$. The Strouhal number becomes moreover independent of the Reynolds number [1, 3-5].

In the case of a two-dimensional flow that is confined between two parallel plates separated by a distance $D$ (see figure 1 , right), the relative confinement of the flow $\left(\frac{d}{D}\right)$ is an additional parameter that may influence the behaviour of the flow. The downstream flow qualitatively exhibits the same phenomena as that observed in the case of a free medium: the wake is still well formed, limited by two streamlines with a constant average pressure and there exists a main frequency. Studies of several researchers show that parameters such as the confinement, the turbulence, the end effects, the geometric shape and the vibration of the bluff body affect quantitatively the overall value of the Strouhal number [6-11]. One question is to identify the choice of typical length scale and velocity scale that would give the simplest expression of a Strouhal number as a function of the Reynolds number and the confinement.

Based on this principle, several techniques have been used for the construction of flowmeters with vortex effect that are different by the way of generating or capturing the 

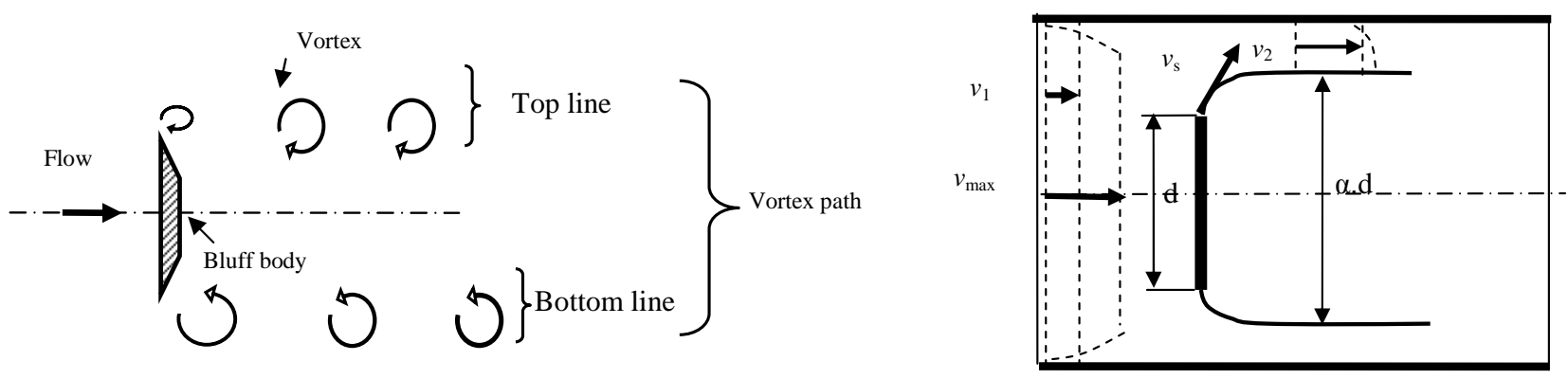

Figure 1: Left: illustration of the vortex path past a bluff body for a flow in infinite medium. Right: sketch of the two-dimensional confined flow between two plates separated by $D$, with a bluff body of cross-section $d$.

eddies. For instance, Sun et al. [12, 13] use a bluff body with sharp edges on which the pressure difference, hence the frequency of vortex shedding, is measured. Peng et al. $[14,15]$ use configurations with two bluff bodies for the generation of vortices. The frequency is determined by measuring fluctuations using two piezoelectric pressure sensors placed at the second hurdle. Hans et al. [16] present a technique that uses a bluff body with a triangular base and two ultrasonic probes for the detection. Bera et al. [17] use a technique called "inductive pick-up" that uses a flexible magnetic strip made of Stainless Steel and placed just downstream of the bluff body to determine the frequency. Takashima et al. [18] have used a bluff body of square section and a double Fiber Bragg grating (FBG) sensor (a technique that uses the interferometric detection to count electromagnetic interference). Miau et al. [19] present in their paper a technique where the bluff body and the sensor are the same piece. A T-shaped bluff body to which is attached a piezoelectric pressure sensor is used, to determine the frequency. We note that in almost all the work presented, the pipe of circular section hinders the formation of vortex roll since the latter is crushed on both ends. Moreover the detection is done at a single point that is quite close to the vortex formation. The results of these studies give the frequency spectrum of vortex shedding, the Strouhal number variation with Reynolds number and the influence of geometry on flow measurement.

The measurement system that is presented thereafter is based on this principle. The originality is to use pipe with square-section in order to get closer to a two-dimensional flow and to separate the generation function from that of detection. Generation is provided by bluff body of trapezoidal section with two parallel sharp edges, behind which vortices develop. The detection is of surface type and is performed at some distance downstream of the obstacle. It is done via a flat plate, parallel to the flow and secured to a beam bending over which are fixed strain gauges. The successive passages of the vortices near the plate stress the beam. The strain gauge transforms the elastic deformation into an electrical signal which, after amplification, accurately reflects the nature of the mechanical vibrations. In principle the number of vortices which are issued and counted during a given time should be directly related to the volumetric flow-rate.

The experimental setup is described in $\S 2$. To evaluate the precision of the apparatus, and to study the effects of the confinement and of the distance between the actuator and the sensor, a specific test loop has been built. The apparatus is first described in $\S 2.1$, and the test loop is described in $\S 2.2$. The accuracy of the measurements is discussed in $\S 2.3$. The results are presented and discussed in $\S 3$ : the search for an optimal position of the bluff body in the pipe and the analysis of the sensitivity of the vortex street to the various geometric parameters is presented in $\S 3.1$ and the effects of the confinement on the calibration of the apparatus are presented in $\S 3.2$. Final remarks and perspectives are then given in $\oint 4$.

\section{Experimental setup and measurement technique}

\subsection{Geometry of the flowmeter}

The technique used in the apparatus shown in figure 2 is based on the generation and detection of vortices with two distinct devices: one for each function. The measurements are performed in a measurement tunnel of square section $D \times D$. The direction of the flow is denoted $x$. A bluff body of cross-section $d \times D$ in the $\{y ; z\}$ plane is secured in the middle of the measurement tunnel (see figure 2, left). This bluff body is of trapezoidal section in the $\{x ; y\}$ plane and thus presents two sharp edges to the flow. The sensor consists of a flat plate parallel to the flow. It is of length $L_{p}$ in the $x$ direction, and placed at a distance $l$ from the bluff body. It is secured to a bending beam over which are fixed strain gauges (see figure 2, right).

\subsection{Description of the dedicated test bench}

The hydraulic system is shown in figure 3 and is designed to cover a range of flow rates from 0 to $50 \mathrm{~m}^{3} / \mathrm{h}$. We show successively:

- a pressurized supply tank (1) with open surface located at a height of $11 \mathrm{~m}$, and of capacity $20 \mathrm{~m}^{3}$; 

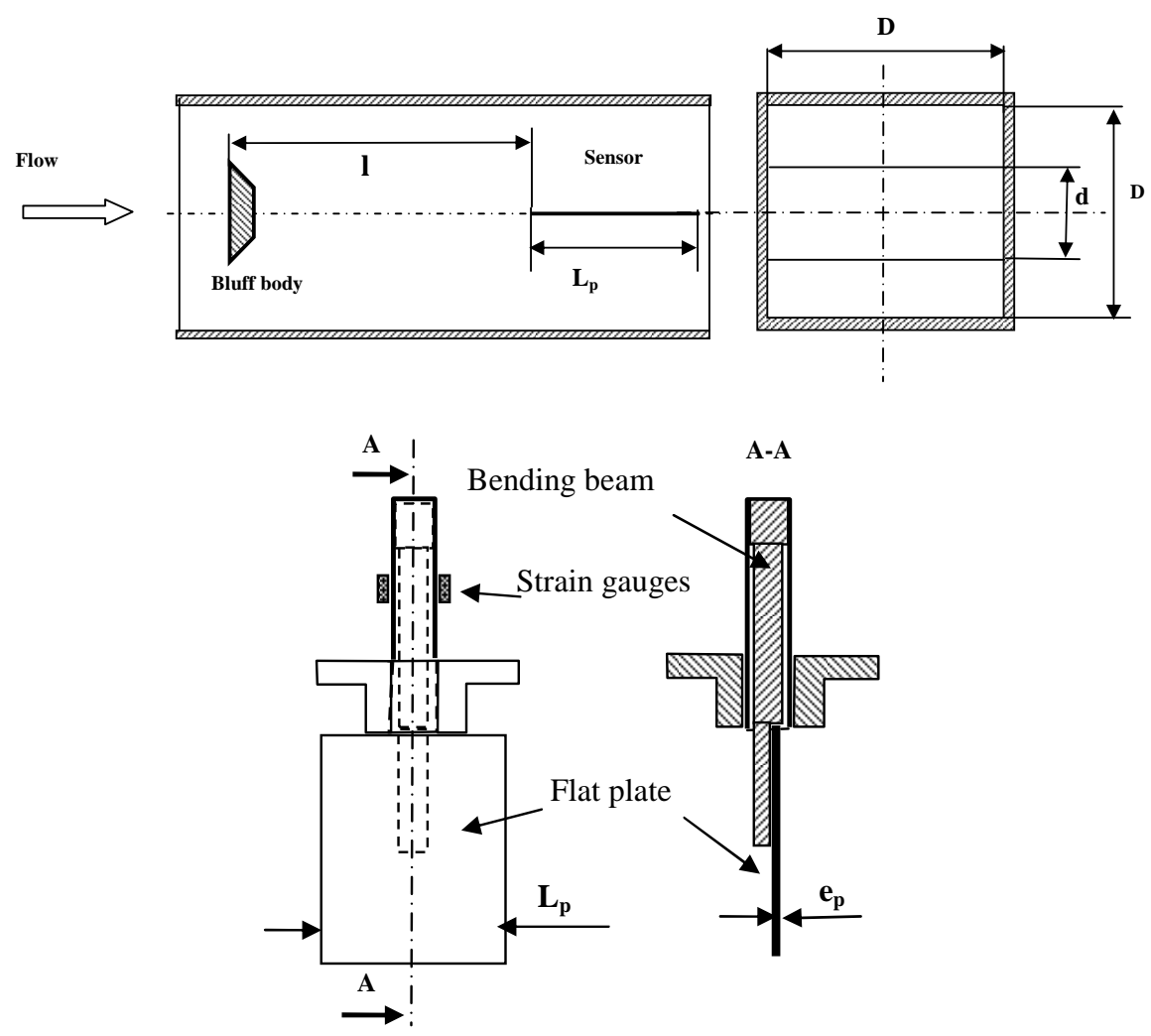

Figure 2: Geometric arrangement for the proposed technique. Details of the bluff body and of the vortex sensor.

- a distribution brass pipe (2) of length $10 \mathrm{~m}$ and inner diameter $200 \mathrm{~mm}$, terminated by a supply valve;

- another line consisting of PVC pipes (3) of diameter $80 \mathrm{~mm}$;

- a testing tunnel (4) which houses the generator and the vortex sensor connected to a measuring system consisting of various devices for measurement, display and acquisition;

- another pipe of diameter $80 \mathrm{~mm}$ made of PVC (5) ending in gooseneck and provided with a valve (6);

- in parallel a flexible hose (7) with a quick release nozzle (8) (similar to gasoline pumps in service stations);

- a receiving gauged tank with a capacity of $1 \mathrm{~m}^{3}(9)$, in parallel with a recovery pit (10) located below the floor;

- a flexible conduit (7) attached to the goose-neck that can guide the jet, either toward the pit or to the gauge volume for measurements;

- a centrifugal pump (11), to pump the fluid between the tank and supply tank.

The measuring system includes the main following components:
- a strain gauge Vishay-micromeasurement which is a Wheatstone bridge specially designed for use with strain gauges operating in bending;

- a computer-amplifier based on the principle of synchronization of a square wave signal with the signal delivered by the strain gauge;

- a passband electronic filter to eliminate the background noise and possible irregularities that can sometimes plague the signal;

- a data acquisition system.

The tests are performed using a protocol that consists of filling the gauged tank by operating at constant average flow, for a given value of the confinement $\frac{d}{D}$ (ratio of characteristic dimensions of the bluff body and testing tunnel). A wide range of flow-rates are tested for each configuration. Several geometrical arrangement of the generator and sensor, i.e. several combinations of $l$ and $L_{p}$, have been tested. The quantities obtained by the measuring devices are: volume $V$ (close to 1000 litres), the number of vortices $N$ (near 4000) and the corresponding time $t$ to fill the gauge.

The calculated quantities are:

- the digital volume, i.e. the volume of fluid passed for every vortex shed $V_{p}=\frac{V}{N}$; 


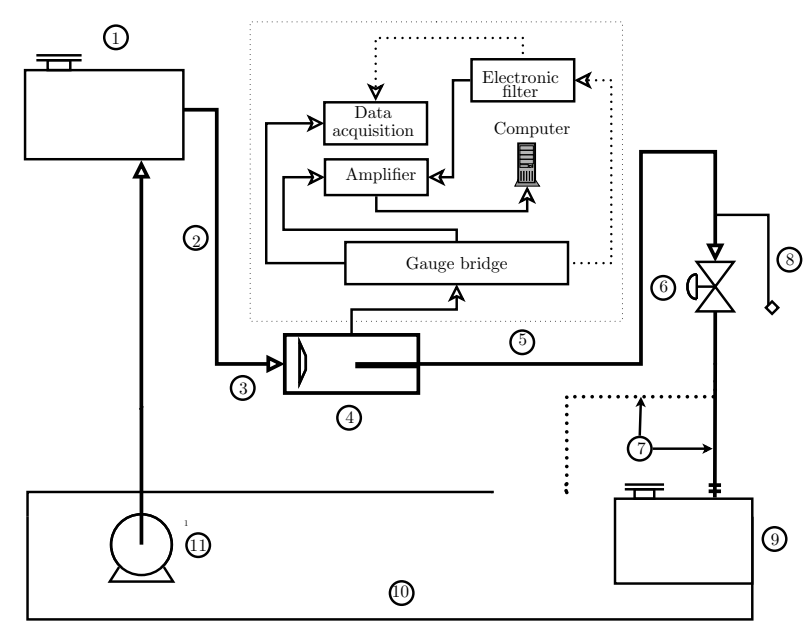

Figure 3: Sketch of the test loop.

- the mean frequency of vortex shedding $f=\frac{N}{t}$;

- the average flow rate $q_{v}=\frac{V}{t}$;

- a Reynolds number based on the characteristic dimension of the testing tunnel $(D)$ and on the discharge velocity:

$$
R e_{D}=\frac{q_{v} D}{A_{t} \nu}
$$

with $A_{t}$ the tunnel cross-section and $\nu$ the kinematic viscosity of water;

- a Reynolds number based on the characteristic dimension of the obstacle generator $(d)$ and on the same velocity scale:

$$
R e_{d}=\frac{q_{v} d}{A_{t} \nu}
$$

- a Strouhal number based on the bluff body characteristic length $d$, and on the same velocity scale:

$$
S_{t}=\frac{f \cdot d}{q_{v} / A_{t}}
$$

- a dimensionless (reduced) digital volume:

$$
V_{r}=\frac{V_{p}}{d \cdot D^{2}} \cdot f(d / D)
$$

with $f(d / D)$ a function that will be introduced thereafter in order to give a universal reduced digital volume that will only depend on the Reynolds number.

\subsection{Accuracy of the measurements}

The objective of this flow-meter is to give a measure of the flow-rate that is as accurate as possible. The typical constancy of the calibration factor for the considered class of flow-meters is of the order of $\pm 0.7 \%- \pm 1 \%$ over a dynamic range of $3-6[1,3,10,13,19]$. In order to find the calibration with a relative precision of let's say $\pm 0.1 \%$ of the measured value, it is first necessary to measure the volume, the number of vortices and the time with a resolution better than $\pm 0.1 \%$.

It it necessary to distinguish between three types of volumes used in the same operation:

1. The volume disposed from the upstream reservoir $V_{1}$ and discharged at the outlet conduit between the first and the last valves.

2. The displayed volume $V_{2}$ of the volume gauge of about 1000 litres. It is known with an uncertainty of reading of $\delta V_{2}=0.25$ litre. This volume is equal to that received by the dry gauge, otherwise it differs by a certain amount $\Delta V_{2}=1$ litre due to the water film wetting the inner wall of the gauge; we have in fact: $V_{1}=V_{2}-\Delta V_{2} \pm \delta V_{2}$. If we suppose that the wettability remains the same, $\Delta V_{2}$ can be regarded as a constant.

3 . The counted volume $V_{3}$ that defines the number of vortices indicated by the electronic counter, we have: $V_{3}=N \cdot V_{p}$. It is not equal to $V_{1}$ for two reasons. The first is that since $N$ is an integer, a mistake of one unit at the beginning and at the end of counting can be made, which results in an uncertainty of about 2 . The second reason is of physical origin and depends on the method used to fill the gauge. We can write $V_{1}=V_{3} \pm(2+\delta N) V_{p}$

It comes as an apparent digital volume:

$$
\frac{V_{2}}{N}=V_{p}\left(1 \pm\left(\frac{2+\delta N}{N}\right)\right)+\frac{\Delta V_{2}}{N} \pm \frac{\delta V_{2}}{N}
$$

Suppose that $\delta N=2$ and that $N \simeq 4000$. For a digital volume to be measured $V_{p}$ of the order of $V_{p} \simeq 0.25$ litre, the maximum relative uncertainty of the digital volume measurement is $2.25 \times 10^{-3}$, but working under the same conditions of wetting of the gauge, the value is reduced to $1.25 \times 10^{-3}$.

The calculation of the characteristic quantities and the corresponding uncertainties give a maximum relative error of the order of $0.4 \%$, so that the relative variations of quantities around their mean value are of the order of $\pm 0.2 \%$. These small variations do not affect the calculation of the average digital volume.

\section{Results}

\subsection{Optimal geometric layout and representative frequency signals}

The desired relative accuracy is of the order of one thousandth of the measured value. The greatest care and every precaution then have to be taken in order to obtain significant repetitive signals. Hundreds of observations of digitized signals have been made and those which seemed 


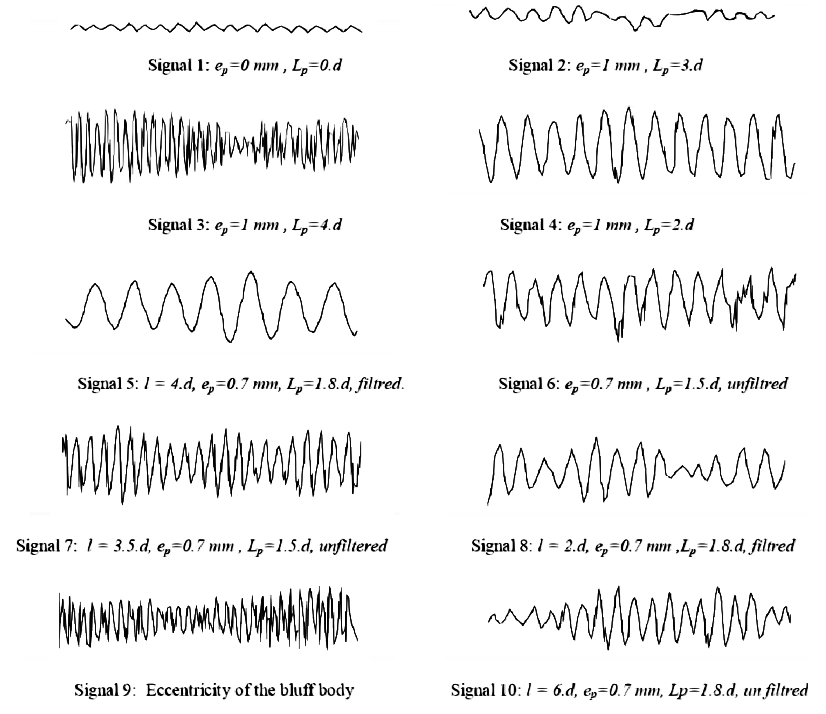

Figure 4: Representative Signals of the shedding vortices frequency.

most significant have been selected and are shown in figure 4 .

The geometric arrangement directly affects the quality of the electric signal collected at the output of the strain gauge. The surface detection type that is used in the present apparatus, in contrast to the volume and point detection types, may result in an easily exploitable signal, providing a good choice of the geometric arrangement. The geometric parameters that can influence the correct operation of the measuring device and thus ensure the accuracy are:

- the width of the sensor plate $\left(L_{p}\right)$;

- the distance between the bluff body and the sensor $(l)$;

- the material and thickness of the plate used to detect vortices $\left(e_{p}\right)$;

- the orientation of the sensor plate with respect to the flow direction;

- the misalignment of the vortices generator relative to the axis of the pipe.

Without entering into the details of the analysis, our goal is to get significant and repetitive signals. Therefore the shape of the signal has been studied for different geometric configurations. An unfiltered signal may result in double counting of vortices (signal 6). Other signals that are not exploitable (signals 1, 2, 3, 8, 9 and 10) are examples of the deleterious effects of the material used for the sensor, the thickness of the plate, a bad balance of the natural bluff body, a very large or very small width of the plate, or a bad plate bluff body.

By optimizing the geometric layout, the search for a significant signal showed that it was entirely possible to have a quasiperiodic signal for all flow rates (signals 4 , 5 and 7 ). For a bluff body of a given dimension, this optimization is characterized by the following parameters:

- a thickness of $e_{p}=0.7 \mathrm{~mm}$ for the plate sensor;

- a width $L_{p}$ of $1.5 d$ to $2 d$ for the plate sensor;

- a bluff body-plate sensor distance $l$ of $3.5 d$ to $4.5 d$.

\subsection{Effects of the geometrical confinement on the digital volume}

In a first step, for a given configuration, tests were performed to see how accurately the digital volume value repeats itself. The results show that if the measurements are repeated with the same conditions, a constant value which reproducibility is better than $10^{-3}$ and a very small variation of the digital volume with the flow rate are found. The values of $V_{p}$ for $d=15 \mathrm{~mm}$, i.e. for a containment of $\frac{d}{D}=0.25$ and for two flow-rates are reported in Tab. 1 . More values are displayed in figure 5 .

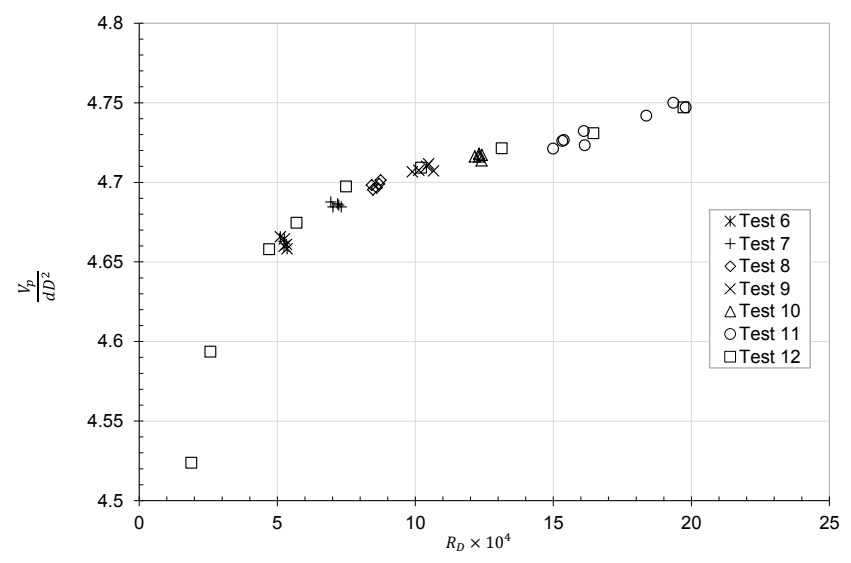

Figure 5: Reproducibility of digital volume changes with the Reynolds number, bluff body characteristic dimension $d=15 \mathrm{~mm}$ (confinement $\frac{d}{D}=0.25$ ).

The figure 5 shows that for a given containment, the digital volume indeed depends on the Reynolds number $R e_{D}$, hence on the flow rate of the pipeline. The digital volume increases with the flow rate: the variation is much stronger at low values of Reynolds number, and one can notice some stabilization for the highest values. Furthermore, the excellent reproducibility of the curve with an accuracy of $\pm 0.1 \%$ is once more verified.

The next step is to find the most significant dimensionless representation of the digital volume that would take into account the effects of the confinement.

The digital volume is:

$$
V_{p}=\frac{V}{N}=\frac{q_{v}}{f}
$$

If we introduce the Strouhal number $S_{t}$, we get:

$$
V_{p}=\frac{D^{2} d}{S_{t}}
$$




\begin{tabular}{|c|c|c|c|c|c|}
\hline Flow rate $q_{v}($ litre/s) & \multicolumn{5}{|c|}{ Digital Volume $V_{p}$ (litres) } \\
\cline { 2 - 6 } & Test 1 & Test 2 & Test 3 & Test 4 & Test 5 \\
\hline 1.56 & 0.24805 & 0.24821 & 0.24814 & 0.24802 & 0.24827 \\
\hline 4.53 & 0.25366 & 0.25385 & 0.25368 & 0.25361 & 0.25381 \\
\hline
\end{tabular}

Table 1: Digital volume $V_{p}$ for $\frac{d}{D}=0.25$.

By dimensional analysis, the Strouhal number is a function of the confinement and of the Reynolds number:

$$
S_{t}=g\left(\frac{d}{D}, R e_{d}\right)
$$

It will be shown in the following that $g\left(\frac{d}{D}, R e_{d}\right)$ can be written as the product of two functions of only $\frac{d}{D}$ and $R e_{d}$, i.e.:

$$
g\left(\frac{d}{D}, R e_{d}\right)=f\left(\frac{d}{D}\right) \cdot h\left(R e_{d}\right)
$$

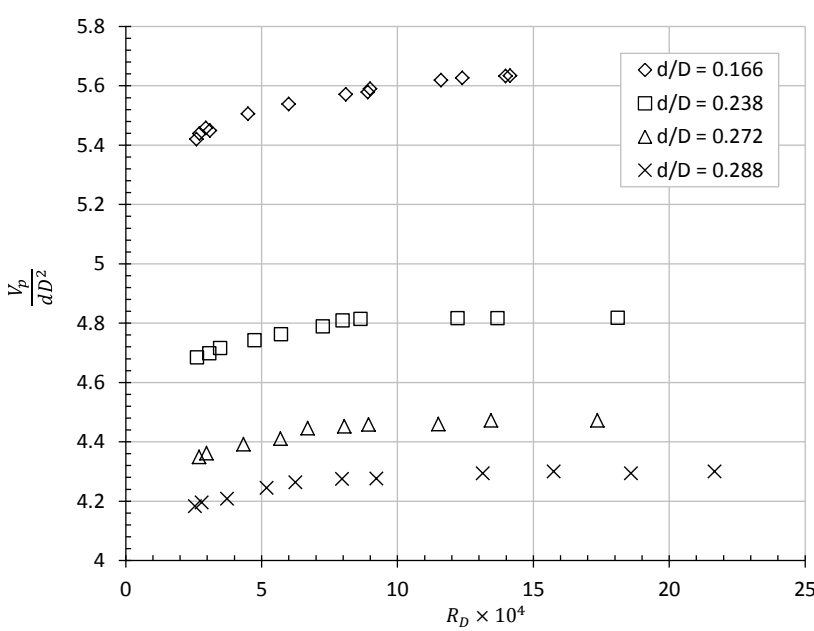

Figure 6: Variation of the dimensionless digital volume with the Reynolds number of the pipe for different confinements.

The variations of $\frac{V_{p}}{d D^{2}}$ - that is thus the inverse of the previously defined Strouhal number $S_{t}$ - with the confinement $\frac{d}{D}$ and the Reynolds number $R e_{d}$ are therefore separately studied in the next paragraphs. The results are plotted in figure 6 , as functions of the Reynolds number for various confinements. The curves look overall the same: there is a very slight increase of the dimensionless digital volume with the Reynolds number. For a fixed confinement, the digital volume varies by roughly $2 \%$ whilst the Reynolds number varies by one order of magnitude. This variation moreover may be well represented by a unique $h\left(R e_{d}\right)$ function with a prefactor depending only on the confinement. For a constant Reynolds number, the digital volume decreases with an increase of the confinement. The dependence with the confinement is more significant: for the two extreme cases that are presented in figure 6 , i.e. $d=0.166$ and 0.288 the variation of the digital volume is roughly of the order of $60 \%$.

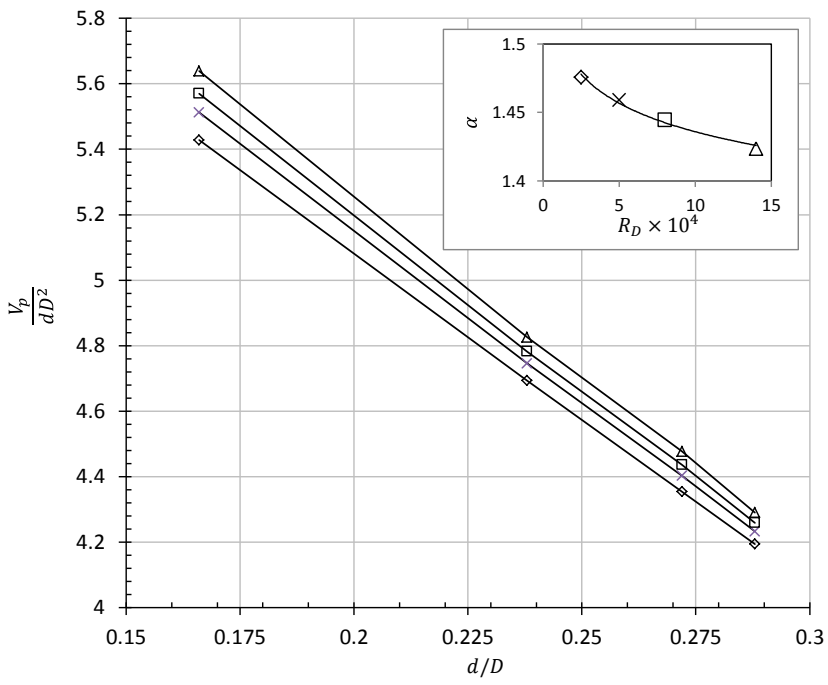

Figure 7: Variation of the dimensionless digital volume with confinements for several Reynolds numbers $\left(R e_{D}\right)$. Inset: parameter $\alpha$ vs. $R e_{D}$, where the parameter $\alpha$ is obtained by a fit of the form $\frac{V_{p}}{d D^{2}}=V_{r}\left(R e_{D}\right) \cdot\left(1-\alpha \frac{d}{D}\right)$.

The dimensionless digital volume $\frac{V_{p}}{d D^{2}}$ as a function of the confinement $\frac{d}{D}$ at different constant Reynolds numbers is plotted in figure 7 . The results show that the function $f\left(\frac{d}{D}\right)$ may be well represented by a function of the form $f(x)=a \cdot x+b$. A simple interpretation can be found if this dependence is written in the equivalent form:

$$
f(x)=V_{r} \cdot(1-\alpha x)
$$

The results show that $\alpha$ only has a very slight dependence with the Reynolds number (see inset in figure 7 ).

The value of $\alpha$ is interpreted as the width of the wake behind the bluff body (see Fig. 1, right). Let us define a second Strouhal number with the velocity of the wake $\left.v_{2}=q_{v}(D-\alpha D)\right)^{-1}$ :

$$
S_{t 2}=\frac{f d}{v_{2}}
$$

Then the "reduced digital volume" is the inverse of this second Strouhal number, that seems to be only a function of the Reynolds number:

$$
V_{r}=\frac{V_{p}}{d D^{2}\left(1-\alpha \frac{d}{D}\right)}
$$

Owing to the negligible dependence of $\alpha$ with $R e_{D}$, a constant value of $\alpha=1.455$ is assumed to compute the 


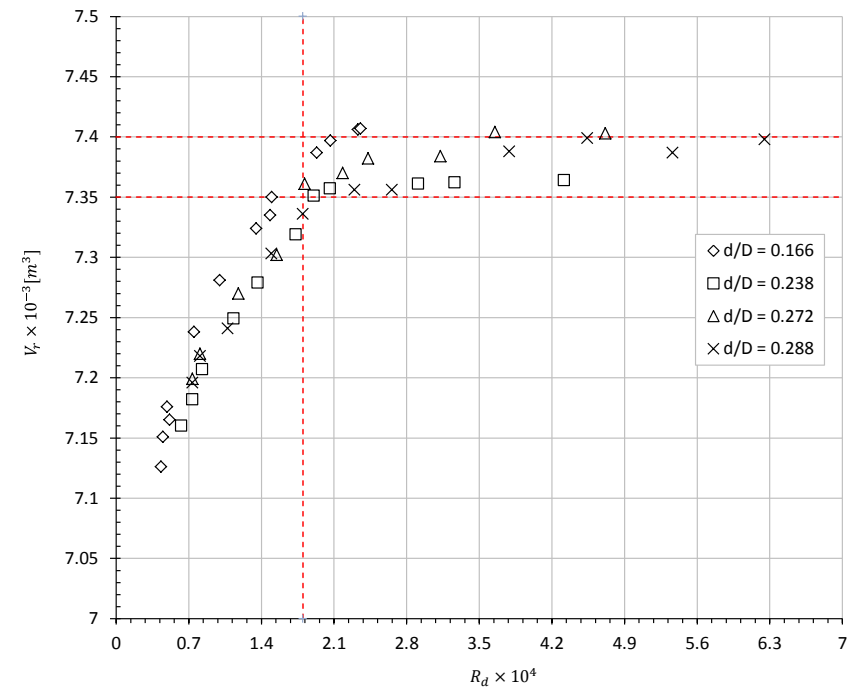

Figure 8: Universal curve (variation of the reduced digital volume).

reduced digital volume. The variation of $V_{r}$ with $R e_{d}$ is plotted in figure 8. All the results are grouped around a universal curve with an accuracy of about $0.3 \%$ : this dimensionless representation shows that the reduced digital volume is independent of the confinement of the fluid stream. The reduced digital volume $V_{r}$ varies slightly at low Reynolds numbers. A plateau is obtained past a threshold in the Reynolds number equal to $1.8 \times 10^{4}$ : a constant value of $V_{r}=7.373$ is obtained with an accuracy of $\pm 3 \times 10^{-3}$, a value slightly lower than the target, in the range $1.8 \times 10^{4} \leq R e_{d} \leq 6.3 \times 10^{4}$, a dynamic range of about 4.5 which is comparable to the state of the art $[1,3,10,13,19]$. The latter might be improved by using a testing tunnel of rectangular section.

\section{Conclusions}

The designed and produced apparatus is suitable for measurements of flow rates averaged for a sufficiently long time. To design and implement an accurate volumetric flow rate measurement unit through the vortex street or the wake generated by a bluff body, an optimal geometrical arrangement of the bluff body, the sensor and the testing tunnel has been suggested that should lead to the best possible signal quality and thus to minimize the errors. The variation of the digital volume with the confinement and with the Reynolds number, led us to define a reduced digital volume $V_{r}$. It is a dimensionless quantity equal to the inverse of the Strouhal number defined with the average velocity in the contracted section downstream of the bluff body. The reduced digital volume has a constant value equal to 7.373. It is a constant of the proposed volume counter. It is represented by a universal curve independent of the geometric parameters. The relative accuracy of our device is about $\pm 0.3 \%$ in a range of flow rates from $3.0 \mathrm{l} / \mathrm{s}$ to $13.3 \mathrm{l} / \mathrm{s}$, which is noteworthy result.

\section{References}

[1] T. Ghaoud and D.W. Clarke. Modelling and tracking a vortex flow-meter signal. Flow Measurement and instrumentation, 13:103-117, 2002.

[2] C.H.K. Williamson and R. Govardhan. A brief review of recent results in vortex-induced vibrations series. Journal of Wind Engineering and Aerodynamics, 96:713-735, 2008.

[3] J.J. Miau, C.W. Wu, C.C. Hu, and J.H. Chou. A study on signal quality of a vortex flow meter downstream of two elbows out-of-plane. Flow Measurement and instrumentation, 13:7585, 2002.

[4] J.P. Bentley and J. Mudd. Vortex shedding mechanisms in single and dual bluff bodies. Flow Measurement and instrumentation, 14:23-31, 2003.

[5] G. L. Pankanin, A. Kulinczak, and J. Berlinski. Investigation of karman vortex street using flow visualization and image processing. Sensors and Actuators, A 138:366-375, 2007.

[6] S. Goujon-Durand. Technical note, linearity of the vortex meter as function of fluid viscosity. Flow Measurement and instrumentation, 6(3):235-238, 1995.

[7] J.P. Bentley, R.A. Benson, and A.J. Shanks. The development of dual bluff body vortex flow meters. Flow Measurement and instrumentation, 7(2):85-90, 1996.

[8] C.H.K. Williamson. A series in $\frac{1}{\sqrt{r_{e}}}$ to represent the strouhalreynolds number relationship of the cylinder wake. Journal of Fluids and Structures, 12:1073-1085, 1998.

[9] J.J. Miau, C.C. Hu, and J.H. Chou. Reponse of a vortex flow meter to impulsive vibrations. Flow Measurement and instrumentation, 11:41-49, 2000.

[10] H. Zhang, Y. Huang, and Z. Sun. A study of mass flow rate measurement based on the vortex shedding principle. Flow Measurement and instrumentation, 17:29-38, 2006.

[11] A. Venugopal, A. Agrawal, and S.V. Prabhu. Influence of blockage and upstream disturbances of the performance of a vortex flowmeter with a trapezoidal bluff body. Measurement, 43(4):603-616, 2010.

[12] Z. Sun, H. Zhang, and J. Zhou. Investigation of the pressure probe properties as the sensor in the vortex flowmeter. Sensors and Actuators, A 136:646-655, 2007.

[13] J. Zhou Z. Sun, H. Zhang. Evaluation of uncertainty in a vortex flowmeter measurement. Measurement, 41:349-356, 2008.

[14] J. Peng, X. Fu, and Y. Chen. Flow measurement by new type vortex flowmeter of dual triangulate bluff body. Sensors and Actuators, A 115:53-59, 2004.

[15] J. Peng, X. Fu, and Y. Chen. Experimental investigations of strouhal number for flows past dual triangulate bluff bodies. Flow Measurement and instrumentation, 19:350-357, 2008.

[16] V. Hans and G. Poppen. Vortex shedding flow meters and ultrasound detect ion: signal processing and influence of bluff body geometry. Flow Measurement and instrumentation, 9:79-82, 1998.

[17] S.C Bera, J.K. Ray, and S. Chattopadhyay. A modified inductive pick-up type technique of measurement in a vortex flow meter. Measurement, 35:19-24, 2004.

[18] S. Takashima, H. Asanuma, and H. Niitsuma. A water flowmeter using dual fiber bragg grattting sensors and cross-correlation technique. Sensors and Actuators, A 116:66-74, 2004.

[19] J.J. Miau, C.F. Yeh, C.C. Hu, and J.H. Chou. On measurement uncertainty of a vortex flow meter. Flow Measurement and instrumentation, 16:397-404, 2005. 


\section{Nomenclature}

Roman characters

\begin{tabular}{|c|c|c|}
\hline$A_{t}$ & {$\left[m^{2}\right]$} & tunnel cross-section \\
\hline$d$ & {$[m]$} & charcteristic dimension of the bluff body \\
\hline$D$ & {$[m]$} & characteristic dimension of the test section \\
\hline$e_{p}$ & {$[m]$} & thickness of the plate sensor \\
\hline$f$ & {$[H z]$} & mean vortex shedding frequency \\
\hline$l$ & {$[m]$} & distance between the vortex generator and the plate sensor \\
\hline$L_{p}$ & {$[m]$} & length of the plate sensor \\
\hline$N$ & {$[-]$} & number of counted vortices \\
\hline$q_{v}$ & {$\left[m^{3} \cdot s^{-1}\right]$} & volumetric flow rate \\
\hline$R e_{d}$ & {$[-]$} & Reynolds number based on $d$ \\
\hline $\operatorname{Re}_{D}$ & {$[-]$} & Reynolds number based on $D$ \\
\hline$S_{t}$ & {$[-]$} & Strouhal number based on $f, d$ and discharge velocity \\
\hline$S_{t 2}$ & {$[-]$} & Strouhal number based on $f, d$ and $v_{2}$ \\
\hline$t$ & {$[s]$} & time to fill the gauge \\
\hline$v_{1}$ & {$\left[m^{3} \cdot s^{-1}\right]$} & free-stream velocity \\
\hline$v_{2}$ & {$\left[m^{3} \cdot s^{-1}\right]$} & velocity of the wake \\
\hline$V$ & {$\left[m^{3}\right]$} & total volume passed through the test section \\
\hline$V_{1}$ & {$\left[m^{3}\right]$} & volume discharged \\
\hline$V_{2}$ & {$\left[m^{3}\right]$} & displayed volume of the volume gauge \\
\hline$V_{3}$ & {$\left[m^{3}\right]$} & counted volume \\
\hline$V_{p}$ & {$\left[m^{3}\right]$} & digital volume \\
\hline$V_{r}$ & {$[-]$} & reduced digital volume \\
\hline
\end{tabular}

Greek characters

$\begin{array}{lll}\alpha & {[-]} & \text { relative width of the wake } \\ \delta V_{2} & {\left[\mathrm{~m}^{3}\right]} & \text { reading uncertainty of } V_{2} \\ \delta N & {[-]} & \text { uncertainty of vortex counting } \\ \Delta V_{2} & {\left[\mathrm{~m}^{3}\right]} & \text { volume loss due to wettability } \\ \nu & {\left[\mathrm{m}^{2} \cdot \mathrm{s}^{-1}\right]} & \text { kinematic viscosity }\end{array}$

